\title{
INTERVENCIÓN EDUCATIVA EN NIÑOS DE ESCUELAS DE TIEMPO COMPLETO EN CIUDAD MANTE, TAMAULIPAS.
}

EDUCATIONAL INTERVENTION IN FULL-TIME SCHOOLS CHILDEN IN CIUDAD MANTE, TAMAULIPAS.

\author{
Sanromán-Martínez María Victoria ${ }^{1}$, Peña-Avelino Luz Yosahandy ${ }^{1}$, Navarro-Álvarez Guadalupe \\ Lorena $^{1,2}$, Rivera-Mellado Milton Carlos ${ }^{3}$, Ceballos-Olvera Ivonne ${ }^{1}$.
}

1 Universidad Autónoma de Tamaulipas. 2 Hospital General de la Zona No. 3. Ciudad de El Mante. 3 Jurisdicción Sanitaria No. VI Ciudad de El Mante, México.

Citation: Sanromán-Martínez M.V., Peña-Avelino L.Y., Navarro-Álvarez G.L., Rivera-Mellado M.C., Ceballos-Olvera I. (2020) Intervención educativa en niños de escuelas de tiempo completo en Ciudad Mante, Tamaulipas. Revista Salud Pública y Nutrición, 19 (4), 1-9.

Editor: Esteban G. Ramos Peña, Dr. CS., Universidad Autónoma de Nuevo León, Facultad de Salud Pública y Nutrición, Monterrey Nuevo León, México. Copyright: (C2020 Sanromán-Martínez M.V., et al. This is an open-access article distributed under the terms of Creative Commons Attribution License [CC BY 4.0], which permits unrestricted use, distribution, and reproduction in any medium, provided the original author and source are credited.

Competing interests: The authors have declared that no competing interests exist.

DOI: https://doi.org/10.29105/respyn19.4-1

Recibido: 09 de julio 2020; $\quad$ Aceptado: 05 de octubre 2020

Email: iceballos@docentes.uat.edu.mx 


\title{
INTERVENCIÓN EDUCATIVA EN NIÑOS DE ESCUELAS DE TIEMPO COMPLETO EN CIUDAD MANTE, TAMAULIPAS.
}

\author{
Sanromán-Martínez María Victoria ${ }^{1}$, Peña-Avelino Luz Yosahandy ${ }^{1}$, Navarro-Álvarez Guadalupe Lorena ${ }^{1,2}$, \\ Rivera-Mellado Milton Carlos ${ }^{3}$, Ceballos-Olvera Ivonne ${ }^{1}$.
}

1 Universidad Autónoma de Tamaulipas. 2 Hospital General de la Zona No. 3. Ciudad de El Mante. 3 Jurisdicción Sanitaria No. VI Ciudad de El Mante, México.

\begin{abstract}
RESUMEN
Introducción. Para prevenir enfermedades relacionadas con desórdenes alimenticios, se deben implementar intervenciones que aborden educación, nutrición y actividad física en escuelas primarias de tiempo completo (EPTC) en México. Objetivo: Evaluar la efectividad de una intervención educativa sobre el estado nutricional, nivel de conocimientos, hábitos de alimentación, actividad física e implementación de menús saludables en escolares de entre 6 y 12 años. Material y Método: Estudio cuasi experimental, descriptivo longitudinal, con una muestra de 262 alumnos de 2 EPTC, (grupo intervenido y grupo control) de Ciudad Mante, Tamaulipas, México. Se realizó una encuesta de conocimiento, frecuencia alimenticia y actividad física, además de la evaluación antropométrica al inicio y al final de la intervención. En el grupo intervenido se cambiaron los menús escolares, se impartieron pláticas sobre los hábitos de alimentación y se implementó actividad física durante cuatro meses. Resultados: El sobrepeso y obesidad general al inicio y final de la intervención fue del $46 \%$ y $28 \%$ respectivamente. La intervención educativa logró disminuir el bajo peso, la obesidad y el sobrepeso. Se incrementó el tiempo de actividad física en los participantes. Conclusiones: La intervención educativa sí es funcional en EPTC con alimentación correcta, hábitos saludables y actividad física puede mejorar el estado nutricional en los escolares.

Palabras Clave: Alimentación, actividad física, sobrepeso y obesidad.
\end{abstract}

\section{ABSTRACT}

Introduction: To prevent eating disorders related to diseases, interventions that address education, nutrition, and physical activity should be implemented in full-time primary schools in Mexico. Objective: To evaluate the effectiveness of an educational intervention on nutritional status, level of knowledge, eating habits, physical activity and healthy menu implementation in schoolchildren between 6 and 12 years old. Material and method: A descriptive, longitudinal, quasiexperimental study with a sample of 262 students from 2 different schools (intervened group and control group) from Ciudad Mante, Tamaulipas, Mexico. Knowledge, frequency of food consumption, and physical activity survey were carried out, in addition to the anthropometric evaluation before and after the intervention. In the intervened group, school menus were changed, talks were given on eating habits, and physical activity was implemented in four months. Results: Overweight and general obesity before and after the intervention were $46 \%$ and $28 \%$, respectively. The educational intervention managed to reduce low weight, obesity, and overweight. Participants' physical activity time was increased. Conclusions: The educational intervention itself is functional in full-time schools with correct nutrition, healthy habits, and physical activity that can improve nutritional status in schoolchildren.

Key words: Nutrition, physical activity, overweight and obesity. 


\section{Introducción}

Se estima que a nivel mundial la obesidad se ha triplicado desde 1975 y para el 2016, había más de 340 millones de niños y adolescentes (entre 5 y 19 años) con sobrepeso u obesidad (NCD-RisC, 2017; OMS, 2020). En América Latina dicha condición se presenta en aproximadamente 42.5 millones. En el caso de México, la ENSANUT (Romero et al., 2013) reportó una prevalencia de sobrepeso y obesidad del $16 \%$ y $15 \%$ respectivamente en niños de 5 a 12 años. Mientras que, en el caso de los adolescentes, esta prevalencia de sobrepeso y obesidad fue de $23 \%$ y 14\% respectivamente (Shamah et al., 2011). Para el 2018 la obesidad en escolares y adolescentes sin ayuda alimentaria aumentó en un $97 \%$ y $60 \%$ respectivamente. El reporte general mostró un aumento de 2.2 veces en la obesidad con respecto a lo reportado en el 2012 (Shamah et al., 2019). En el 2012, Tamaulipas reportó una prevalencia de sobrepeso y obesidad de 18.7 y $23.7 \%$ en escolares (Rivera et al., 2013). La Organización de las Naciones Unidas (OMS) para la Alimentación y la Agricultura (FAO, 2018) indicó que la tendencia a incrementar la obesidad y el sobrepeso es consecuencia de diferentes factores entre los que destacan: crisis económicas, conflictos sociales, cambio climático y principalmente cambios en los hábitos alimentarios. El estado nutricional de las personas está asociado a la condición física y es el balance entre consumo y gasto energético (Martínez, 2014). Un desequilibrio en el estado nutricional, pueden originar desórdenes alimenticios como: la desnutrición, la obesidad, las enfermedades crónicas degenerativas y las deficiencias vitamínicas (Casanueva et al., 2001). La educación nutricional y el incremento en la actividad física repercute en la nutrición, pues al mejorar el comportamiento dietético y el estado de nutrición en los niños, se evitará a largo plazo enfermedades aún en presencia de antecedentes genéticos (FAO, 2018). Algunas estrategias que se han implementado para la disminución del sobrepeso y la obesidad en escolares son las intervenciones integrales (desde educativas hasta la implementación de actividad física) que han mostrado reducción en el índice de masa corporal (IMC), menor consumo de alimentos no saludables y aumento de conocimientos sobre salud nutricional (Kain et al., 2012; Ratner et al., 2013; Vio et al., 2014). En México se han implementado una serie de intervenciones, en las cuales se han observado cambios en los estilos de vida promoviendo la disminución del IMC (Alvirde et al., 2013), incremento en la selección y consumo de alimentos saludables (Safdie et al., 2013; Vargas, 2019), y aumento en la actividad física (Gatica et al., 2019). No obstante, en México se ha demostrado la presencia incrementada del sobrepeso y obesidad (Romero et al., 2013; Shamah et al., 2019). La evidencia disponible sobre intervenciones integrales que evalúen estrategias combinadas para prevenir el sobrepeso y la obesidad en escolares aún es insuficiente. Una intervención educativa que promueva hábitos saludables ayudara a disminuir el sobrepeso y la obesidad en escolares.

En el 2007, en México, el Programa de Escuelas de Tiempo Completo comenzó con 500 escuelas ubicadas en 15 estados mexicanos y para el año escolar 2012-2013, ya había 6,715 escuelas en los 32 estados federales mexicanos (Zermeño et al., 2014). En el año 2018, en Tamaulipas, ya existían 860 escuelas de tiempo completo. En el caso de El Mante, Tamaulipas, se tenían registradas 95 escuelas (10 privadas y 85 públicas) con una población total 11,006 alumnos inscritos en el nivel primaria, de las cuales 14 escuelas eran de tiempo completo (SEP, 2020). El Programa, propuso dar atención a la problemática de alimentación ya que, en estos planteles, se cuenta con un espacio para el refrigerio y se ha establecido así la enseñanza de hábitos y valores en el fomento de la convivencia armónica y la conservación de la salud. Una de las recomendaciones fue planificar el menú diario con productos de temporada y los de consumo tradicional en la comunidad, incluyendo los tres grupos de alimentos: frutas y verduras, cereales y tubérculos, y leguminosas y alimentos de origen animal. Sin embargo, en dichos planteles no se cuenta con especialistas en nutrición, por lo tanto, el objetivo del presente trabajo es evaluar la efectividad de una intervención educativa sobre el estado nutricional, nivel de conocimientos, la importancia de los buenos hábitos de alimentación y actividad física en escuelas primarias de tiempo completo mediante la participación de un especialista en nutrición, chef y activador físico.

\section{Material y Método}

Se realizó un estudio cuasi experimental, descriptivo, analítico y longitudinal, llevado a cabo de enero a mayo del 2018, cuya población objetivo fue de 262 niños de dos escuelas primarias de tiempo completo 
(en horario de 8 a 16 horas) de edades comprendidas entre 6 y 12 años, que acudían a clases en la primaria "Ricardo J. Zevada" grupo intervenido (131) y como grupo control (131) alumnos de la escuela "Club de Leones". Ambas instituciones contaron aproximadamente con una población total de 211 alumnos. Estas escuelas se localizan en Ciudad Mante Tamaulipas, México. Los padres de familia o tutores y alumnos fueron informados sobre el estudio. Los responsables del cuidado de los menores firmaron un consentimiento informado autorizando la participación de sus hijos en el estudio en ambas instituciones (control e intervenida). Los criterios de inclusión considerados fueron: alumnos inscritos en el plantel educativo, madres de familia y docentes que presentaron consentimiento informado firmado, y que participaron en los talleres, mientras que los criterios de exclusión fueron niños, madres de familia y docentes que no quisieran participar en el proyecto y los alumnos inscritos que no se encontraron al momento de la evaluación inicial. criterios de eliminación: alumnos que no acudieron a todas las sesiones educativas y que no realizaron las evaluaciones finales, madres de familia o docentes que no asistieron a las sesiones educativas.

Una vez obtenido el consentimiento informado, se aplicó una encuesta sobre los hábitos de alimentación y ejercicio a los alumnos de ambas instituciones educativas. La encuesta aplicada se basó en el instrumento previamente validado en Chile con algunas adaptaciones (Lera et al., 2015). para determinar el estado nutricional de los alumnos participantes antes y después de la intervención se realizó un tamizaje de peso, talla e IMC de acuerdo con lo propuesto por OMS, 2020 y la NOM-008 SSA-1993, comparando con las tablas de percentiles de 5 a 19 años. En la escuela intervenida se realizaron 9 sesiones educativas para los niños, con una duración de 20 minutos cada una [hábitos de alimentación (porciones correctas de acuerdo a la edad los niños, Plato de bien comer, importancia de agua natural, importancia de las frutas y verduras en cada tiempo de comida, la importancia de la actividad física y disminución del uso de aparatos electrónico como entretenimiento)] y tres talleres dos para padres el primero con el tema de alimentos saludables, porciones, preparación y cocción; el segundo taller a los padres sobre los efectos negativos de malos hábitos de alimentación en los niños, la actividad física) uno curso taller para docentes (actividad física diaria en los niños), lo anterior con el fin de que los padres estuvieran informados sobre estos temas. El taller realizado con los profesores, fue organizado para la implementación de la actividad física con los niños la cual se realizó de lunes a viernes durante 15 minutos antes de entrar a clases, incluyendo ejercicios de estiramiento y coordinación con música. Se realizó la revisión del menú de desayunos escolares; este análisis reveló un contenido alto de energía (1545 kcal/d, entre el desayuno y la comida), $37.7 \mathrm{~g}$ de proteínas, lípidos $74.1 \mathrm{~g}$ e hidratos de carbono $185.3 \mathrm{~g}$ y se implementaron nuevos menús en la escuela intervenida con variedad de alimentos, porciones correctas, mejor aporte nutritivo y balance energético por edad, basada en una dieta estandarizada de $1500 \mathrm{kcal}$ por día, proteína $56 \mathrm{~g}$, lípidos $50 \mathrm{~g}$, hidratos de carbono 206.2. En ambos grupos control e intervenido la encuesta y tamizaje de medidas antropométricas se realizó antes y después de la intervención.

Los resultados de la encuesta, el peso, estatura, IMC y diagnóstico nutricional fueron capturados en el programa de Excel 2010, Microsoft Office. Para la obtención de porcentajes, se construyeron tablas de contingencia por género y estado nutricional; además de los cambios de hábitos de actividad física. Se realizó una prueba $\mathrm{t}$ de Student para muestras dependientes del IMC y para las dimensiones asociadas a los hábitos, consumo de alimentos y actividad física Los datos fueron analizados en el programa IBM Statistical Package for the Social Sciences (SPSS) Statistics Software v25.

\section{Resultados}

El presente estudio se llevó a cabo con la participación de 262 alumnos de los cuales fueron 74 hombres y 57 mujeres entre 6 y 12 años de edad (muestra $=131)$ pertenecientes a la escuela primaria "Ricardo J. Zevada" como grupo intervenido. Los alumnos de la escuela primaria "Club de Leones" como grupo control fueron 73 hombres y 58 mujeres de 6 a 12 años de edad Ambas escuelas son de tiempo completo donde los niños realizan dos comidas durante el horario escolar.

En la tabla 1, se muestran los resultados del estado nutricional (bajo, normal, sobrepeso y obesidad) al inicio y final de la intervención en función del género y del grupo evaluado (intervenido $\mathrm{n}=131$ y control 
$\mathrm{n}=131$ ). En el grupo de niños intervenidos se observó $3.8 \%$ de bajo peso, $49.5 \%$ peso normal, $21.3 \%$ sobrepeso y $25.1 \%$ obesidad antes de la intervención. Después de la intervención, no se observaron niños con bajo peso, en peso normal fue de $71.7 \%$, en sobrepeso $9.2 \%$ y obesidad $19 \%$. Estos resultados indican que el estado nutricional normal mejoró en un $22 \%$ en niños que participaron en la intervención. El estado nutricional del grupo control fue el siguiente: bajo peso de $9.9 \%$, peso normal de $28.9 \%$, sobrepeso en $10.6 \%$ y obesidad $50.3 \%$; después de la intervención se mostró los siguientes resultados: el peso bajo fue de $0.7 \%$, el peso normal de $44.2 \%$, el sobrepeso $18.2 \%$ y la obesidad $36.5 \%$. En el grupo control hubo un incremento del $15.3 \%$ en estado nutricional normal. Es importante resaltar que el sobrepeso y obesidad se presentó con mayor frecuencia en los niños comparado con las niñas que participaron en este estudio. Estas condiciones en el grupo de estudio disminuyeron un $18.2 \%$, comparado con el grupo control el cual solo disminuyó un $6.2 \%$. Sin embargo, en los valores promedio de IMC no se encontró diferencia significativa $\left(p>0.05^{*}\right)$ entre los grupos estudio y control (tabla 2).

\begin{tabular}{cccccc}
\multicolumn{7}{c}{ intervención } \\
\hline \multirow{2}{*}{$\begin{array}{c}\text { Estado } \\
\text { nutricional }\end{array}$} & $\begin{array}{c}\text { Fase de la } \\
\text { intervención }\end{array}$ & $\begin{array}{c}\text { Intervenido } \\
\mathrm{n}(\%)\end{array}$ & $\begin{array}{c}\text { Control } \mathrm{n} \\
(\%)\end{array}$ & $\begin{array}{c}\text { Femenino } \\
\text { Intervenido } \\
\mathrm{n}(\%)\end{array}$ & $\begin{array}{c}\text { Control } \mathrm{n} \\
(\%)\end{array}$ \\
\hline \multirow{2}{*}{ Bajo } & Inicial & $2(1.5)$ & $7(5.3)$ & $3(2.3)$ & $6(4.6)$ \\
& Final & $0(0)$ & $1(0.7)$ & $0(0)$ & $0(0)$ \\
\multirow{2}{*}{ Normal } & Inicial & $40(30.5)$ & $23(17.5)$ & $25(19.0)$ & $15(11.4)$ \\
& Final & $52(39.7)$ & $32(24.4)$ & $42(32)$ & $26(19.8)$ \\
\multirow{2}{*}{ Sobrepeso } & Inicial & $15(11.4)$ & $7(5.3)$ & $13(9.9)$ & $7(5.3)$ \\
& Final & $9(6.9)$ & $9(6.8)$ & $3(2.3)$ & $15(11.4)$ \\
\multirow{2}{*}{ Obesidad } & Inicial & $17(12.9)$ & $36(27.4)$ & $16(12.2)$ & $30(22.9)$ \\
& Final & $13(9.9)$ & $31(23.6)$ & $12(9.1)$ & $17(12.9)$ \\
\hline
\end{tabular}

boración propia

$*_{n}=262$

Tabla 2. Prueba t de Student para muestras dependientes del IMC con relación al género, durante la pre y post-intervención.

\begin{tabular}{|c|c|c|c|c|c|}
\hline \multirow[b]{2}{*}{ Grupo } & \multirow[b]{2}{*}{ Género } & \multicolumn{2}{|c|}{ Pre-intervención } & \multicolumn{2}{|c|}{ Pos-intervención } \\
\hline & & $\mathrm{X}$ & $\mathrm{DE}$ & 又 & $\mathrm{DE}$ \\
\hline \multirow{2}{*}{ Estudio } & Masculino & 18.51 & 4.52 & 17.94 & 4.14 \\
\hline & Femenino & 19.1 & 5.52 & 17.8 & 3.45 \\
\hline \multirow{2}{*}{ Control } & Masculino & 19.23 & 4.96 & 19.61 & 4.82 \\
\hline & Femenino & 18.87 & 3.78 & 19.12 & 3.85 \\
\hline
\end{tabular}

Fuente: Elaboración propia

En la tabla 3, de acuerdo a la cantidad de respuestas por niño se analizaron las siguientes tres dimensiones: frecuencia de consumo de alimentos, hábitos y actividad física. Los resultados reflejan una diferencia significativa $\left(\mathrm{p}<0.0001^{* *}\right)$ en la frecuencia de consumo y hábitos en los niños del grupo intervenido. No se presentaron diferencias en ninguna dimensión en el grupo control. Lo anterior, sugiere que los niños en el grupo intervención consumieron en menor frecuencia alimentos de alto aporte calórico. Los niños no dejaron de comer solo modificaron lo que comieron aumentando la frecuencia de consumo de verduras, frutas, agua y colaciones sanas en la semana. El conocimiento adquirido se ve reflejando en la elección de alimentos saludables, así como la frecuencia de consumo figura 1. No se observaron cambios respecto a la actividad física.

\begin{tabular}{|c|c|c|c|c|c|}
\hline \multirow[b]{2}{*}{ imensión } & \multirow[b]{2}{*}{ Grupo } & \multicolumn{2}{|c|}{$\begin{array}{l}\text { Pre- } \\
\text { intervención }\end{array}$} & \multicolumn{2}{|c|}{$\begin{array}{l}\text { Pos- } \\
\text { intervención }\end{array}$} \\
\hline & & 又 & $\mathrm{DE}$ & 又 & $\mathrm{DE}$ \\
\hline recuencia de consumo & Estudio* & 2.63 & 0.5 & 2.36 & 0.52 \\
\hline de alimentos & Control & 2.55 & 2.4 & 2.44 & 0.64 \\
\hline \multirow{3}{*}{ Hábitos } & Estudio * & 2.37 & 0.5 & 2.64 & 0.52 \\
\hline & Control & 2.58 & 0.5 & 2.48 & 0.64 \\
\hline & Estudio & 2.46 & 0.6 & 2.47 & 0.6 \\
\hline Actividad física & Control & 2.51 & 0.6 & 2.51 & 0.57 \\
\hline
\end{tabular}

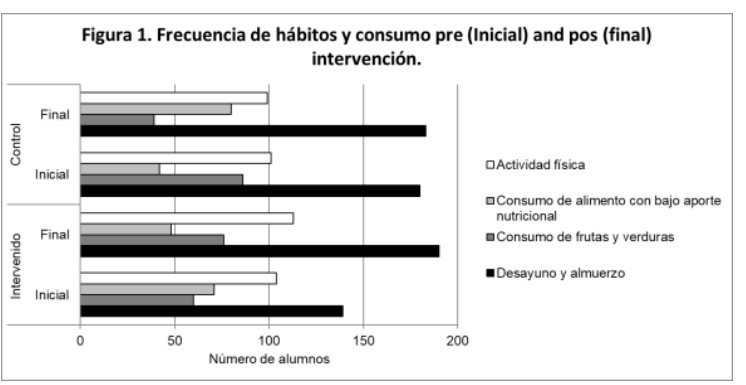

Sin embargo, En la tabla 4 se observa que las frecuencias de respuestas de los alumnos muestran un número mayor para el grupo control en cuanto a la actividad deportiva fuera de la escuela y la disminución del tiempo de uso de algún aparato electrónico (televisión, computadora y consolas de video). 
Tabla 4. Frecuencia de hábitos de actividad física

\begin{tabular}{|c|c|c|c|c|c|c|c|c|}
\hline \multirow[b]{3}{*}{ Frecuencia } & \multicolumn{4}{|c|}{ Intervención } & \multicolumn{4}{|c|}{ Control } \\
\hline & \multicolumn{2}{|c|}{ Inicial } & \multicolumn{2}{|c|}{ Final } & \multicolumn{2}{|c|}{ Inicial } & \multicolumn{2}{|c|}{ Final } \\
\hline & $\mathrm{n}$ & $\%$ & $\mathrm{n}$ & $\%$ & $\mathrm{n}$ & $\%$ & $\mathrm{n}$ & $\%$ \\
\hline \multicolumn{9}{|c|}{ Frecuencia participas en actividades deportivas* } \\
\hline Nunca & 27 & 20.6 & 18 & 13.7 & 30 & 22.9 & 32 & 24.4 \\
\hline Una vez por semana & 65 & 49.6 & 61 & 46.6 & 40 & 30.5 & 69 & 52.7 \\
\hline $2-3$ veces por semana & 39 & 29.8 & 30 & 22.9 & 34 & 26.0 & 23 & 17.6 \\
\hline Casi todos los días & 0 & 0.0 & 22 & 16.8 & 27 & 20.6 & 7 & 5.3 \\
\hline \multicolumn{9}{|c|}{ Frecuencia participas en actividades recreativas al aire libre* } \\
\hline Nunca & 21 & 16.0 & 19 & 14.5 & 28 & 21.4 & 33 & 25.2 \\
\hline 1 vez por semana & 38 & 29.0 & 48 & 36.6 & 39 & 29.8 & 36 & 27.5 \\
\hline $2-3$ veces por semana & 35 & 26.7 & 26 & 19.8 & 34 & 26.0 & 36 & 27.5 \\
\hline casi todos los días & 37 & 28.2 & 38 & 29.0 & 30 & 22.9 & 26 & 19.8 \\
\hline \multicolumn{9}{|c|}{ Tiempo que realiza actividad física } \\
\hline 20 minutos & 39 & 29.8 & 41 & 31.3 & 37 & 28.2 & 18 & 13.7 \\
\hline 1 hora & 55 & 42.0 & 50 & 38.2 & 44 & 33.6 & 33 & 25.2 \\
\hline 2 horas & 23 & 17.6 & 28 & 21.4 & 31 & 23.7 & 35 & 26.7 \\
\hline No hago & 14 & 10.7 & 12 & 9.2 & 19 & 14.5 & 45 & 34.4 \\
\hline \multicolumn{9}{|c|}{ Horas diarias frente a televisión, computadora, consolas de video juegos } \\
\hline 1 hora & 54 & 41.2 & 69 & 52.7 & 28 & 21.4 & 28 & 21.4 \\
\hline 2 horas & 35 & 26.7 & 28 & 21.4 & 31 & 23.7 & 22 & 16.8 \\
\hline 3 horas & 23 & 17.6 & 17 & 13.0 & 21 & 16.0 & 24 & 18.3 \\
\hline 4 horas o más & 19 & 14.5 & 17 & 13.0 & 51 & 38.9 & 57 & 43.5 \\
\hline
\end{tabular}

La tabla 5 muestra el análisis nutrimental del menú (desayuno y comida) que preparaban las madres de familia antes de la intervención. Este análisis reveló un contenido alto de energía (1545 kcal/d, entre el desayuno y la comida), $37.7 \mathrm{~g}$ de proteínas, lípidos $74.1 \mathrm{~g}$ e hidratos de carbono $185.3 \mathrm{~g}$. Durante la intervención se cambiaron los menús con un menor aporte de energía $(1,195.9 \mathrm{kcal} / \mathrm{d})$ y macronutrientes (37.7 g de proteínas, lípidos $50.5 \mathrm{~g}$ e hidratos de carbono $147.4 \mathrm{~g}$ ). La diferencia en las kilocalorías que no consumieron diariamente en el desayuno y la comida fue de 349.1. Asimismo, el consumo de lípidos fue menor en $23.6 \mathrm{~g}$ y $37.9 \mathrm{~g}$ de hidratos de carbono.

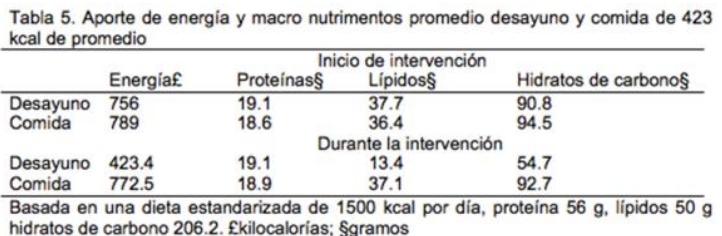

\section{Discusión}

Este estudio mostró una reducción de sobrepeso y obesidad de los niños en la intervención del $18.25 \%$ comparado con los niños del grupo control 6.2\%. de acuerdo con estos resultados Pérez et al. (2008) implementaron un programa educativo en 121 alumnos, con una duración 16 semanas, en el cual se observó disminución del sobrepeso y obesidad, pero no observaron disminución en el IMC. Harris et al. (2009) Aguilar et al. (2011) y Lobos et al. (2013) revelan que la implementación de programas de educación nutricional acoplados a actividad física ayuda a mantener los IMC o reducirlos paulatinamente en periodos de 4 a 12 meses. Tomando en cuenta lo anterior, los programas de educación con mayor duración tendrán mejores resultados en la disminución de IMC, sobrepeso y obesidad en los niños. Estudios realizados por la ENSANUT (Romero et al. 2013) indican que el sobrepeso y la obesidad se presenta en niños $(36.9 \%)$ y niñas (32\%), estos datos coinciden con lo reportado en este estudio y con lo reportado por Ratner et al. (2013) en Santiago de Chile, junto Benítez et al. (2016) en Tepic, Nayarit y Aparco et al. (2017) en Lima.

El grupo de niños intervenido obtuvo un mejor estado nutricional porcentual en comparación con el grupo sin intervención. La diferencia fue de $6 \%$ de incremento en estado condición normal siendo esto posible debido a la modificación de los menús durante la intervención incluyendo las sesiones de activación física. La modificación en un menor consumo de energía y macro nutrimentos de alta densidad calórica, lograron la mejoría en el estatus nutricional. Quizán et al. (2014) emplearon un programa educativo que promueve la alimentación saludable, en este estudio se describen resultados positivos debido a que los participantes en su programa disminuyeron la ingesta de energía (grasas totales y carbohidratos). Los resultados de la presente intervención también guardan relación con lo reportado por Lobos et al. (2013) y Scruzzi et al. (2014) quienes observaron una relación entre la mejora del estado nutricional, la práctica nutricional, y de la actividad física en los niños de primaria. Además, se debe considerar que el tiempo de implementación, y el seguimiento en un programa de intervención es un factor relevante en el éxito de este tipo de programas (López et al., 2010).

El grupo de alumnos intervenidos mostró un cambio de hábitos positivo al consumir alimentos nutritivos (frutas y verduras) en comparación con el grupo 
control. Esto concuerda con el estudio realizado por Santos et al. (2015) en alumnos de diferentes escuelas del estado de Quintana Roo, en el que se demostró un aumento en el consumo de alimentos saludables; así mismo Vio et al. (2014), reportaron la disminución del consumo de alimentos no nutritivos y aumentando el consumo de alimentos nutritivos dentro de la escuela. Romero et al. (2013) reportó que los niños mexicanos consumen preferentemente productos lácteos, antojitos (preparados con grasa proteínas y cereales), bebidas azucaradas pastelillos y galletas. Lo anterior demuestra que la intervención influenció a los niños a consumir alimentos saludables.

Con respecto a la actividad física, este estudio observó una disminución en el sedentarismo en los niños que participaron en la intervención, aumentando la actividad física dentro y fuera de la escuela. Llargués et al. (2012), concluye que el aumento en frecuencias de la actividad física en la escuela, mejora el estado nutricional de los alumnos. El hábito de uso de aparatos electrónicos, como televisión, computadora y video juegos fue menor en el grupo estudio que el grupo control, siendo similar al obtenido por Quizán et al. (2014) quienes mostraron que los niños intervenidos reducen el número de horas dedicadas a actividades sedentarias en comparación con los no intervenidos. Por ello las pláticas de concientización que promueva una mayor activación física también tendrán efecto en el estado nutricio.

En el estudio, se analizó el contenido nutricional del menú preparado en la escuela por las madres de familia, antes de la intervención, donde dicho menú fue sustituido cada semana por uno saludable, logrando así una disminución del aporte energético, lo que ayudó a la mejora del estado nutricional. Actualmente, hay estudios del refrigerio que venden en la escuela y los cambios a lo largo del tiempo (Santos et al., 2015), sin embargo, no hay investigaciones donde se modifiquen los menús en las escuelas de tiempo completo con la intervención de un nutriólogo, sin embargo, la ENSANUT (Romero et al., 2013), reportó un cambio importante en el consumo de colaciones nutritivas y en la frecuencia de los alimentos.

Con respecto a los conocimientos de los hábitos de alimentación correcta, se incrementaron al término de la intervención, sin embargo, los resultados alcanzado no corresponde con los obtenido por Llargués et al. (2012). Lo anterior puede deberse a el tiempo de duración de cada estudio. Esto sugiere que los conocimientos y los hábitos de alimentación correctos requieren de programas de intervención constantes o de mayor duración para logra el impacto deseado.

\section{Conclusiones}

Al implementar una intervención educativa integral que incluya pláticas y talleres sobre alimentación y los efectos nocivos del uso de aparatos electrónicos como entretenimiento y la incorporación de la actividad física. Se logra disminuir la frecuencia de consumo de alimentos con bajo contenido nutricional, se mejoran los hábitos tanto alimenticios como de actividad física en escolares. Además, la implementación de menús en las escuelas realizados por un especialista en nutrición promueve el consumo de alimentos adecuados con los requerimientos energéticos que favorecen el estado nutricional de los niños al disminuir el bajo peso, la obesidad y el sobrepeso. Se sugiere que la intervención se realice durante un tiempo mayor a cuatro meses y se hagan visitas de seguimiento.

\section{Agradecimientos}

La realización del presente trabajo fue gracias a los alumnos participantes de las escuelas "Ricardo J. Zevada" como grupo intervenido y la escuela "Club de Leones" como grupo control. De la misma forma, queremos agradecer a los padres de familia por su valiosa colaboración, y autorización para la participación de sus hijos y, no menos importante damos las gracias, a las direcciones de los centros educativos participantes, por la entusiasta participación durante el tiempo de ejecución de la intervención.

\section{Bibliografía}

Aguilar, M. J., González, E., García, C. J., García, P. A., Álvarez, J., Padilla, C. A., González, J. L., \& Ocete, E. (2011). Obesity in a school children population from Granada: Assessment of the efficacy of an educational intervention. Nutrición Hospitalaria. 26(3), 636-641. https://doi.org/10.3305/nh.2011.26.3.5195

Alvirde, G. U., Rodríguez, G. A. J., Henao, M. S., Gómez, P. F., \& Agular, S. C. (2013). Resultados 
de un programa comunitario de intervención en el estilo de vida en niños Ulices. Salud Pública de México, 55(3), s406-s414.

Aparco, J. P., Bautista, W., \& Pillaca, J. (2017). Impact evaluation of educational-motivational intervention "como jugando" to prevent obesity in school children of cercado de lima: Results in the first year. Revista Peruana de Medicina Experimental y Salud Publica, 34(3), 386-394. https://doi.org/10.17843/rpmesp.2017.343.2472

Benítez, V., De Jesús Vázquez, I., Sánchez, R., Velasco, R., Ruiz, S., De, M., \& Medina, J. (2016). Investigación Intervención educativa en el estado nutricional y conocimiento sobre alimentación y actividad física en escolares. Revista de Enfermería Del Instituto Mexicano Del Seguro Social, 24(1), 37-43.

Casanueva, E., Kaufer k., Pérez. A., \& Arroyo P. (2001). Nutriología Médica. Editorial Médica Panamericana. Buenos Aires-Bogotá-CaracasMadrid-México. pp. 719.

Gatica, G., Moreno, J. E., Cortés, J. D., Henao, S. A., \& Rivera, J. A. (2019). Condición física de escolares tras intervención educativa para prevenir obesidad infantil en Morelos, México. Salud Pública de México, 61, 78-85.

Harris, K. C., Kuramoto, L. K., Schulzer, M., \& Retallack, J. E. (2009). Effect of school-based physical activity interventions on body mass index in children: A meta-analysis. CMAJ, $180(7)$, 719-726. https://doi.org/10.1503/cmaj.080966

Kain, J., Leyton, B., Concha, F., Weisstaub, G., Lobos, L., Bustos, N., \& Vio, F. (2012). [Evaluation of an obesity prevention intervention which included nutrition education and physical activity applied in public schools of Santiago, Chile]. Archivos Latinoamericanos de Nutrición. 62(1), 60-67. http://www.ncbi.nlm.nih.gov/pubmed/23477209

Lera, L., Fretes G., González, C. G., Salinas, J., \& Vio, F. (2015). Validación de un instrumento para evaluar consumo, hábitos y prácticas alimentarias en escolares de 8 a 11 años. Nutrición
Hospitalaria, $\quad 31(5), \quad$ 1977-1988. https://doi.org/10.3305/nh.2015.31.5.8607

Llargués, E., Recasens, A., Franco, R., Nadal, A., Vila, M., Pérez, M. J., Recasens, I., Salvador, G., Serra, J., Roure, E., \& Castell, C. (2012). Evaluación a medio plazo de una intervención educativa en hábitos alimentarios y de actividad física en escolares: Estudio Avall 2. Endocrinología y Nutrición, 59(5), 288-295. https://doi.org/10.1016/j.endonu.2012.03.002

Lobos, L., Leyton, B., Kain-Bercovich, J., \& Vio del Río, F. (2013). Evaluación de una intervención educativa para la prevención de la obesidad infantil en escuelas básicas de Chile. Nutrición Hospitalaria, 28(4), 1156-1164. https://doi.org/10.3305/nh.2013.28.4.6588

López, L., Audisio, Y., \& Berra, S. (2010). Effectiveness of population-based interventions on the prevention of overweight in children and adolescents. Medicina Clínica, 135(10), 462469.

https://doi.org/10.1016/j.medcli.2009.06.010

Martínez, J. A. (2014). Nutrición saludable frente a la obesidad: bases científicas y aspectos dietéticos. Editorial Médica Panamericana.

Non-Communicable Diseases Risk Factor Collaboration, (NCD-RisC). (2017). Worldwide trends in body-mass index, underweight, overweight, and obesity from 1975 to 2016: a pooled analysis of 2416 population-based measurement studies in 128.9 million children, adolescents, and adults. Lancet, 390(10113), 2627-2642

https://www.thelancet.com/action/showPdf?pii= S0140-6736\%2817\%2932129-3

NOM-008-SSA2-1993. (1993). Control de la nutrición, crecimiento y desarrollo del niño y del adolescente. Criterios y procedimientos para la prestación del servicio. http://www.salud.gob.mx/unidades/cdi/nom/008 ssa23.html.

Organización de las Naciones Unidas para la Alimentación y la Agricultura, (FAO). (2018). El estado de la seguridad alimentaria y la nutrición 
en el mundo. Fomentando la resiliencia climática en aras de la seguridad alimentaria y la nutrición. In Organización de las Naciones Unidas para la Alimentación y la Agricultura. http://www.fao.org/3/a-I7695s.pdf

Organización Mundial de la Salud, (OMS), (2020). Obesidad y sobrepeso. Organización Mundial de La Salud. https://www.who.int/es/newsroom/fact-sheets/detail/obesity-and-overweight

Pérez, P., Raigada, J., Collins, A., Mauricio, S., Felices, A., Jiménez, S., \& Casas-Castañeda, J. (2008). Efectividad de un programa educativo en estilos de vida saludables sobre la reducción de sobrepeso y obesidad en el Colegio Effectiveness of an educational program focusing in healthy lifestyles for overweight and obesity reduction in. Acta Medica Peruana, 25(4), 6.

Quizán, T., Meneses, L. V., Romero, J. E., Villar, A. V. B., \& Díaz, R. (2014). Programa educativo afecta positivamente el consumo de grasa, frutas, verduras y actividad física en escolares mexicanos. Nutrición Hospitalaria, 30(3), 552561. https://doi.org/10.3305/nh.2014.30.3.7438

Ratner, R. G., Durán, A. S., Garrido, L. M. J., Balmaceda, H. S., Jadue, H. L., \& Atalah, S. E. (2013). Impacto de una intervención en alimentación y actividad física sobre la prevalencia de obesidad en escolares. Nutrición Hospitalaria, 28(5), 1508-1514. https://doi.org/10.3305/nh.2013.28.5.6644

Rivera, J., Cuevas, L., Shamah, T., Valenzuela, D. G., \& Ávila, M. A. (2013). Encuesta Nacional de Salud y Nutrición 2012 Resultados por Entidad Federativa. Instituto Nacional de Salud Pública, 168-171.

Romero, M., Shamah, T., Franco, A., Villalpando, S., Cuevas, L., Gutiérrez, J. P., \& Rivera, J. Á. (2013). Encuesta nacional de salud y nutrición 2012: Diseño y cobertura. Salud Pública de México, 55(SUPPL.2), 332-340. https://doi.org/10.21149/spm.v55s2.5132

Safdie, M., Jennings, N., Lévesque, L., Janssen, I., Campirano, F., López, N., Aburto, T., \& Rivera, J. A. (2013). Impact of a school-based intervention program on obesity risk factors in Mexican children. Salud Publica de México, 55(SUPPL.3).

https://doi.org/10.21149/spm.v55s3.5138

Santos, M. de los A. A., Fernández, T. del N. J. G., Rodríguez, G. V., Campo, C. S. M. del, \& Gómez, A. M. (2015). Intervención educativa para la mejora de prácticas de alimentación y actividad física en escuelas primarias del estado de Quintana Roo. Revista Española de Nutrición Comunitaria, 21(4), 9-16. https://doi.org/10.14642/RENC.2015.21.4.5117

Scruzzi, G., Cebreiro, C., Pou, S., \& Rodríguez, C. (2014). Salud escolar: una intervención educativa en nutrición desde un enfoque integral. Cuadernos. Info, 35(35), 39-53. https://doi.org/10.7764/cdi.35.644

Secretaria de Educación Pública, (SEP). (2020). Anuario de la estadística educativa de Tamaulipas. Secretaria de Educación. https://www.tamaulipas.gob.mx/educacion/basic a/

Shamah, T., Campos, I., Cuevas, L., Hernández, L., Morales, M., Rivera, J., \& Barquera, S. (2019). Sobrepeso y obesidad en población mexicana en condición de vulnerabilidad. Resultados de la Ensanut. Salud Publica México, 61, 852-865.

Shamah, T., Cuevas, L., Méndez, I., Jiménez, A., Mendoza, A. J., \& Villalpando, S. (2011). La obesidad en niños mexicanos en edad escolar se asocia con el consumo de alimentos fuera del hogar: durante el trayecto de la casa a la escuela. Archivos Latinoamericanos de Nutrición, 61(3), 288-295.

Vargas, M. C. A. (2019). Evaluación de la mejora del conocimiento de los escolares que cursan tercer y cuarto grado de primaria sobre "El Plato del Bien Comer" después de la intervención educativa en nutrición. JÓVENES EN LA CIENCIA, 6.

Vio, F., Salinas, J., Montenegro, E., González, C. G., \& Lera, L. (2014). Efecto de una intervención educativa en alimentación saludable en profesores y niños preescolares y escolares de la 
región de Valparaíso, Chile. Nutrición Hospitalaria, 29(6), 1298-1304. https://doi.org/10.3305/nh.2014.29.6.7409

Zermeño, G. M. G., Fahara, M. F., \& Garza, L. A. de la. (2014). The full-time school program in Mexico. Journal of Case Studies in Education, 115. 